\title{
Kondo effect and singlet-triplet splitting in coupled quantum dots in a magnetic field
}

\author{
Vitaly N. Golovach and Daniel Loss \\ Department of Physics and Astronomy, University of Basel, Klingelbergstrasse 82, CH-4056 Basel, Switzerland
}

(November 2, 2018)

\begin{abstract}
We study two tunnel-coupled quantum dots each with a spin $1 / 2$ and attached to leads in the Coulomb blockade regime. We study the interplay between Kondo correlations and the singlettriplet exchange splitting $K$ between the two spins. We calculate the cotunneling current with elastic and inelastic contributions and its renormalization due to Kondo correlations, away and at the degeneracy point $K=0$. We show that these Kondo correlations induce pronounced peaks in the conductance as function of magnetic field $B$, inter-dot coupling $t_{0}$, and temperature. Moreover, the long-range part of the Coulomb interaction becomes visibile due to Kondo correlations resulting in an additional peak in the conductance vs $t_{0}$ with a strong $B$-field dependence. These conductance peaks thus provide direct experimental access to $K$, and thus to a crucial control parameter for spin-based qubits and entanglement.
\end{abstract}

Semiconductor quantum dots have attracted much interest over the years due to their rich and reproducible transport properties in the Coulomb blockade (CB) regime, where the number of electrons on the dot is quantized due to charging effects [1]. More recently, attention has focused on the spin of the electron in such nanostructures, with the spin introducing new correlation effects such as Kondo behavior [2 6], and its interplay with spin exchange interaction in single [7 10 and double dots 11, 12]. On the other hand, it has been pointed out that the spin, confined to a quantum dot or atom, is a suitable candidate for quantum information processing [13], due to unusually long decoherence time of spin 14]. A crucial element in such spin-based quantum computing schemes is the Heisenberg exchange interaction $K$ (singlet-triplet splitting) between spins of adjacent dots, being controlled via the interdot tunneling [13]. Thus, the primary goal is to achieve control over $K$ which then allows one to generate deterministic entanglement of spins. Using a Hund-Mulliken (HM) approach to describe a realistic double dot system (DD) it has been shown [15] that $K$ is very sensitive to long range Coulomb interaction as well as to magnetic fields by which a singlet-triplet crossing can be tuned. Motivated by this we study here transport and Kondo effects in such a realistic DD system within the HM approach, thereby going beyond short-range on-site models used so far to describe Kondo effects. In particular, we calculate the current through the DD via a Schrieffer-Wolff transformation and via a systematic cotunneling calculation including elastic and inelastic contributions. Using a perturbative renormalization group (RG) approach we show that the conductance in the cotunneling regime shows pronounced peaks induced by Kondo correlations and long range Coulomb interactions as function of temperature, inter-dot coupling, magnetic fields, and bias. Such Kondo enhanced peaks in the conductance thus provide direct experimental access to singlet/triplet states and their exchange splitting $K$-the quantities of crucial importance for spin-based qubit schemes.
We consider a DD system consisting of two lateral quantum dots tunnel-coupled to metallic leads, in the presence of a perpendicular magnetic field $B$, see Fig. 1 . At low temperatures $T$, the conductance $G$ of the DD as a function of the gate voltage $V_{g}$ shows sharp doublets of sequential tunneling peaks separated by $\mathrm{CB}$ valleys (cotunneling regime) [16]. In the middle between such peaks, the number of electrons in the DD is even, $M=2 N$ (assuming similar dots). We assume that $N-1$ electrons of each dot form a closed shell [with $N-1$ even] and thus ignore them. The remaining two electrons in the DD can be described by the Hamiltonian

$$
H_{d}=\sum_{i=1,2}\left[\frac{1}{2 m}\left(\mathbf{p}_{i}-\frac{e}{c} \mathbf{A}\left(\mathbf{r}_{i}\right)\right)^{2}+W\left(\mathbf{r}_{i}\right)\right]+C,
$$

where $C=e^{2} / \kappa\left|\mathbf{r}_{\mathbf{1}}-\mathbf{r}_{\mathbf{2}}\right|$ is the Coulomb interaction, with charge $e$ and dielectric constant $\kappa(=13.1$ for GaAs). As usual in GaAs, the Zeeman interaction will be neglected.

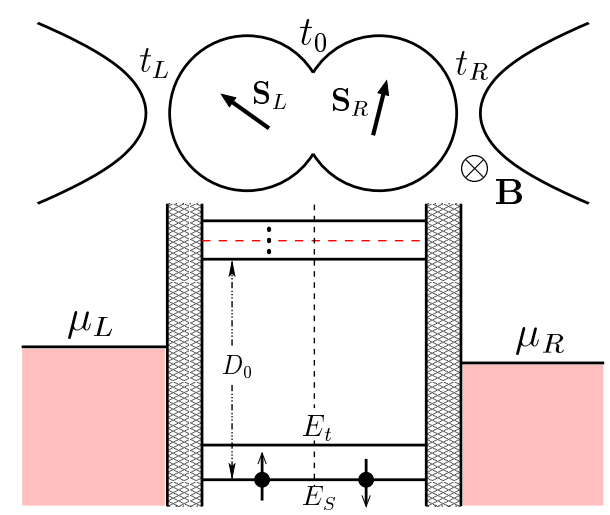

FIG. 1. Double-dot system containing two electrons and being coupled in series to two metallic leads at chemical potentials $\mu_{R}$ and $\mu_{L}$ with bias $\Delta \mu=\mu_{L}-\mu_{R}$. The electron spins $\mathbf{S}_{L}, \mathbf{S}_{R}$ interact via the exchange interaction $K=E_{t}-E_{S}$, where $E_{t, S}$ is the triplet/singlet energy.

However, the orbital effect of the magnetic field $\mathbf{B}=$ $\nabla \times \mathbf{A}$ is important, it allows us to tune the singlet-triplet 
transition in the isolated DD, provided, however, there is long-range Coulomb interaction [15]. Within the low energy sector, a realistic confinement potential for the DD is given by 15] $W(\mathbf{r})=\left(m \omega_{0}^{2} / 2\right)\left[\left(x^{2}-a^{2}\right)^{2} / 4 a^{2}+y^{2}\right]$, which separates at $a \gg \sqrt{\hbar / m \omega_{0}}$ into two harmonic wells of frequency $\omega_{0}$, located at $x= \pm a$, with, typically in GaAs, $h \omega_{0} \simeq 3 \mathrm{meV}$ [1]. Within the HM approach we express the two-particle states of the DD [15] in terms of symmetric (for spin singlet) and antisymmetric (for spin triplet) combinations of the Fock-Darwin states, given by $\varphi_{ \pm a}(x, y)=\exp \left[-\left((x \mp a)^{2}+y^{2}\right) / 2 \lambda^{2} \mp i y a / 2 l^{2}\right] / \lambda \sqrt{\pi}$, where $l=\sqrt{\hbar c /|e| B}$ and $\lambda=\sqrt{\hbar / m \omega}$, and $\omega=$ $\sqrt{\omega_{0}^{2}+\omega_{L}^{2}}$, where $\omega_{L}=|e| B / 2 m c$. We introduce the operators $d_{ \pm, \sigma}^{\dagger}\left(d_{ \pm, \sigma}\right)$, which create (annihilate) the DD states $\psi_{ \pm, \sigma}=\chi_{\sigma}\left(\varphi_{-a} \pm \varphi_{+a}\right) / \sqrt{2(1 \pm S)}$, where $\chi_{\sigma}$ is the spinor and $S=\left\langle\varphi_{ \pm a} \mid \varphi_{\mp a}\right\rangle$ the overlap integral. The singlet and triplet states then become,

$$
\begin{aligned}
& |00\rangle=\frac{1}{\sqrt{1+\phi^{2}}}\left(d_{+\uparrow}^{\dagger} d_{+\downarrow}^{\dagger}-\phi d_{-\uparrow}^{\dagger} d_{-\downarrow}^{\dagger}\right)|0\rangle, \\
& |11\rangle=d_{-\uparrow}^{\dagger} d_{+\uparrow}^{\dagger}|0\rangle, \quad|1-1\rangle=d_{-\downarrow}^{\dagger} d_{+\downarrow}^{\dagger}|0\rangle, \\
& |10\rangle=\frac{1}{\sqrt{2}}\left(d_{-\uparrow}^{\dagger} d_{+\downarrow}^{\dagger}+d_{-\downarrow}^{\dagger} d_{+\uparrow}^{\dagger}\right)|0\rangle,
\end{aligned}
$$

where $|0\rangle$ is the DD ground state with $M=2 N-2$, and

$$
\phi=\sqrt{1+\left(\frac{4 t_{H}}{U_{H}}\right)^{2}}-\frac{4 t_{H}}{U_{H}},
$$

where $t_{H}=t_{0}+t_{C}$ is the extended inter-dot tunneling amplitude with $t_{0}$ its bare value $(C=0)$ and $t_{C}\left(t_{0}\right)$ the part that gets renormalized by the Coulomb interaction $C$. Similarly, $U_{H}$ is the extended on-site Coulomb repulsion which decreases with decreasing a [15]. Note that for $t_{0}=0$ we have $\phi=1$, while $\phi<1$ occurs due to double occupancies in the dots, and $\phi \rightarrow 0$ for $C \rightarrow 0$. The DD is connected to two Fermi liquid leads $\alpha=L, R$ (Fig. 1), described by $H_{l}=\sum_{\alpha k \sigma} \varepsilon_{k} c_{\alpha k \sigma}^{\dagger} c_{\alpha k \sigma}$, where $c_{\alpha k \sigma}^{\dagger}$ creates an electron-state with momentum $k$ and spin $\sigma$ in lead $\alpha$. The tunnel coupling from lead $\alpha$ to the nearest dot is parametrized by the amplitude $t_{\alpha}$, and thus the amplitude to tunnel from lead $\alpha$ into the DD states $\psi_{n \sigma}$ is then given by $t_{L, \pm}=t_{L} / \sqrt{2(1 \pm S)}$, $t_{R, \pm}= \pm t_{R} / \sqrt{2(1 \pm S)}$. We use the tunneling Hamiltonian $H_{T}=\sum_{n \alpha k \sigma}\left(t_{\alpha n} c_{\alpha k \sigma}^{\dagger} d_{n \sigma}+\right.$ h.c. $)$ to perform a Schrieffer-Wolff transformation [17], and arrive at the cotunneling part $H_{c o t}=H_{T}\left[\left(i 0^{+}-\hat{L}_{0}\right)^{-1} H_{T}\right]$, with Liouvillean $L_{0} A \equiv\left[H_{0}, A\right]$, where $H_{0}=H_{d}+H_{l}$.

Next we concentrate on the states (2) and project out the higher lying energy levels by means of the mapping 8

$$
\begin{aligned}
& \sum_{\sigma \sigma^{\prime}} d_{n \sigma}^{\dagger} \vec{\sigma}_{\sigma \sigma^{\prime}} d_{n^{\prime} \sigma^{\prime}}=\mathbf{S}_{+} \delta_{n n^{\prime}}+\left(\frac{\phi_{+}}{2} \mathbf{S}_{-}+i n \phi_{-} \mathbf{T}\right) \delta_{-n n^{\prime}} \\
& \sum_{\sigma} d_{n \sigma}^{\dagger} d_{n^{\prime} \sigma}=\delta_{n n^{\prime}}\left[1-\frac{n}{2} \phi_{+} \phi_{-}\left(\mathbf{S}_{L} \cdot \mathbf{S}_{R}-\frac{1}{4}\right)\right]
\end{aligned}
$$

where $\mathbf{S}_{ \pm}=\mathbf{S}_{L} \pm \mathbf{S}_{R}, \mathbf{T}=\mathbf{S}_{L} \times \mathbf{S}_{R}$, and $\phi_{ \pm}=\sqrt{2}(1 \pm$ $\phi) /\left(1+\phi^{2}\right)$. The spin $1 / 2$ operators $\mathbf{S}_{L, R}$ represent the two electron spins on the DD [15]. We arrive at the effective Hamiltonian

$$
\begin{aligned}
H= & H_{l}+K \mathbf{S}_{L} \cdot \mathbf{S}_{R}+\Delta H \\
\Delta H= & \sum_{\alpha \alpha^{\prime}}\left(J_{\alpha \alpha^{\prime}} \mathbf{s}_{\alpha \alpha^{\prime}} \cdot \mathbf{S}_{+}-V_{\alpha \alpha^{\prime}} \rho_{\alpha \alpha^{\prime}} \mathbf{S}_{L} \cdot \mathbf{S}_{R}\right. \\
& \left.+I_{\alpha \alpha^{\prime}}^{+} \mathbf{s}_{\alpha \alpha^{\prime}} \cdot \mathbf{S}_{-}+2 i I_{\alpha \alpha^{\prime}}^{-} \mathbf{s}_{\alpha \alpha^{\prime}} \cdot \mathbf{T}\right),
\end{aligned}
$$

where $\mathbf{s}_{\alpha \alpha^{\prime}}=\sum_{k k^{\prime} \sigma \sigma^{\prime}} c_{\alpha k \sigma}^{\dagger}\left(\vec{\sigma}_{\sigma \sigma^{\prime}} / 2\right) c_{\alpha^{\prime} k^{\prime} \sigma^{\prime}}$, and $\rho_{\alpha \alpha^{\prime}}=$ $\sum_{k k^{\prime} \sigma} c_{\alpha k \sigma}^{\dagger} c_{\alpha^{\prime} k^{\prime} \sigma}$. In (5) the bare constants $J, V, I^{ \pm}$are defined at the energy cutoff $D_{0} \simeq \hbar \omega_{0}$,

$$
J=\frac{2}{E_{C}} V^{+}, \quad V=\frac{\phi_{+} \phi_{-}}{2 E_{C}} V^{-}, \quad I^{ \pm}=\frac{\phi_{ \pm}}{E_{C}} W^{ \pm},
$$

with matrix elements $V_{\alpha \alpha^{\prime}}^{ \pm}=t_{\alpha^{\prime},+}^{*} t_{\alpha,+} \pm t_{\alpha^{\prime},-}^{*} t_{\alpha,-}$, $W_{\alpha \alpha^{\prime}}^{ \pm}=t_{\alpha^{\prime},-}^{*} t_{\alpha,+} \pm t_{\alpha^{\prime},+}^{*} t_{\alpha,-}$, and $E_{C}=2 E_{+} E_{-} /\left(E_{+}+\right.$ $\left.E_{-}\right)$. Here, $E_{ \pm}=E(M \pm 1)-E(M) \mp \mu$ is the CB addition/extraction energy, with $E(M)$ being the energy of the DD with $M$ electrons, and $\mu=\left(\mu_{L}+\mu_{R}\right) / 2$. We calculate $E(M)$ for $M=1,2,3$ within the HM method.

Attaching leads to the DD results in a shift of the DD spectrum such that $K=K_{0}+\delta K_{S W}$, where

$$
K_{0}=\mathrm{v}-\frac{U_{H}}{2}+\frac{1}{2} \sqrt{U_{H}^{2}+16 t_{H}^{2}}
$$

is the exchange interaction of the isolated DD, with $\mathrm{v}$ accounting for long range Coulomb effects [15], and $\delta K_{S W}=2 \nu D_{0}\left(E_{C} / E_{-}\right) \operatorname{Tr} V<0$ stems from the Schrieffer-Wolff transformation. We next derive the RG equations for the coupling constants in (6) by applying the "poor man's" scaling approach 18. In matrix form the RG equations read,

$$
\begin{aligned}
& \dot{J}=J^{2}+\left(I^{+}\right)^{2}-\left(I^{-}\right)^{2}, \quad \dot{V}=2\left[I^{-}, I^{+}\right], \\
& \dot{I}^{ \pm}=\left\{J, I^{ \pm}\right\}+\left[V, I^{\mp}\right],
\end{aligned}
$$

where the dot denotes $d / d(\nu \mathcal{L})$. Here $\nu$ is the density of states per spin in the leads, and $\mathcal{L}=\ln \left(D_{0} / D\right)$, with $D$ being the scaled cutoff. Eqs. (9) are valid for $D \gg$ $K, \Delta \mu, T_{0}$ (for $T_{0}$ see below). The set of 16 equations (9) can be reduced to 9 equations for our case of $t_{\alpha n}$ being real 19. Solving (9) numerically we find the characteristic energy scale of the problem, $T_{0}=D_{0} \exp \left(-\gamma / \nu J_{0}\right)$, where $J_{0}=\left(t_{L}^{2}+t_{R}^{2}\right) / E_{C}$, and $\gamma \leq 0.5$ is a non-universal number, determined by the ratios of the coupling constants (7) to $J_{0}$, and thus depends on the internal features of the DD. We note that at $t_{R}=0$ we find $\gamma=\left(1-S^{2}\right) / 4$, i.e. independent of the Coulomb interaction. Also, in the limit of separated dots, $t_{0}=0$, Eqs. (9) decouple into two RG equations, determining the Kondo temperatures for two $1 / 2$ spins [18], $T_{K(L, R)}^{0}=D_{0} \exp \left(-E_{C} / 4 t_{L, R}^{2}\right)$.

We derived Eqs. (9) assuming a singlet-triplet degeneracy (STD) of the DD, i.e. $K=0$. However, the $\mathrm{RG}$ 
procedure generates terms that also renormalize the exchange $K$ between the DD spins. Indeed, we find

$$
\dot{K}=2 \ln (2) D \nu^{2} \operatorname{Tr}\left\{V^{2}+\left(I^{+}\right)^{2}-\left(I^{-}\right)^{2}-J^{2}\right\} .
$$

The RG flow (10) resembles the generation of the RKKY interaction in the two-impurity Kondo model 20]. It follows from (10), (9) that, at low $T \sim T_{0}$, the renormalized $K=K(\bar{B}, D)$ strongly depends on both $D$ and $B$ at the STD point $B_{*}$ with $K\left(B_{*}, D\right)=0$, see right inset of Fig. 3. This suggests that, (i) $B_{*}$ shifts towards the triplet side as $T$ is lowered down to $T_{0}$, (ii) the energy scale of the problem strongly depends on $K_{0}$, which presumably implies a rather sharp crossover between a spin 1 Kondo regime on the triplet side and a locked singlet of $\mathbf{S}_{L}, \mathbf{S}_{R}$ (i.e. no Kondo effect) on the singlet side.

We perform a non-equilibrium calculation of the current through the DD up to the second order in the perturbation (6). The current consists of an elastic and inelastic component, $\mathcal{I}_{K}=\mathcal{I}_{\text {el }}+\mathcal{I}_{\text {inel }}$,

$$
\begin{gathered}
\mathcal{I}_{\text {el }}=\frac{e}{\hbar} \pi \nu^{2}\left[J_{L R}^{2}\left\langle\mathbf{S}_{+}^{2}\right\rangle+V_{L R}^{2}\left(\frac{9}{4}-\left\langle\mathbf{S}_{+}^{2}\right\rangle\right)\right] \Delta \mu, \\
\mathcal{I}_{\text {inel }}=\frac{e}{\hbar \beta} \pi \nu^{2}\left(I_{L R}^{-}\right)^{2} g(\beta K) f(\beta K, \beta \Delta \mu),
\end{gathered}
$$

where $\beta=1 / T$ (with Boltzman constant set to one), $g(u)=\left(e^{u}+1\right) /\left(e^{u} / 3+1\right)$ accounts for the degeneracy of the excited level, $\left\langle\mathbf{S}_{+}^{2}\right\rangle=6 /\left(e^{\beta K}+3\right)$, and

$$
f(u, v)=\frac{u \tanh (u / 2) \sinh (v)+v[1-\cosh (v)]}{\cosh (u)-\cosh (v)} .
$$

Eqs. (11), (12), together with the RG equations (9), describe the renormalization of the current $\mathcal{I}, i$. e. $\mathcal{I} \propto$ $\mathcal{I}_{K}\left(\ln \left(D_{0} / T\right)\right)$. However, not all terms were retained in (5), but only those which become renormalized. To give a complete description of $\mathcal{I}$ at temperatures $T_{K} \ll T \leq$ $D_{0}$, we perform a systematic calculation of the cotunneling current using the technique developed in [21]. In the cotunneling regime, including level shifts but neglecting heating effects [21], we match the two currents and obtain for the total current (after lengthy calculation) $\mathcal{I}(\mathcal{L})=\mathcal{I}_{K}(\mathcal{L})+\delta \mathcal{I}(0)$, with

$$
\begin{aligned}
& \delta \mathcal{I}=\frac{e}{\hbar} \pi \nu^{2}\left[V_{L R}^{2}\left(\frac{7}{4}-\left\langle\mathbf{S}_{+}^{2}\right\rangle\right)+J_{L R}^{2}\left(\frac{E_{-}-E_{+}}{E_{-}+E_{+}}\right)^{2}-\right. \\
& \left.2 V_{L R} J_{L R} \frac{E_{-}-E_{+}}{E_{-}+E_{+}}\left(2-\left\langle\mathbf{S}_{+}^{2}\right\rangle\right)\right] \Delta \mu
\end{aligned}
$$

In the cotunneling regime, the differential conductance $G=e d \mathcal{I} / d \Delta \mu$ shows a step at $\Delta \mu= \pm K$, for $T<|K|$. This step is due to the inelastic current (12) contributing for $|\Delta \mu| \geq|K|$. The step height of $G$ is different for the ground state being a singlet or triplet; the ratio of heights is given by $g(K / T)$. As $T$ is lowered, Kondo correlations are expected to develop at $\Delta \mu= \pm K$, with a Kondo peak arising at the step of $G$. Kondo correlations at finite bias are not the subject of the present paper, but we would like to point out that the step in $G$ can be used for determining the ground state of the DD and measuring $K$.

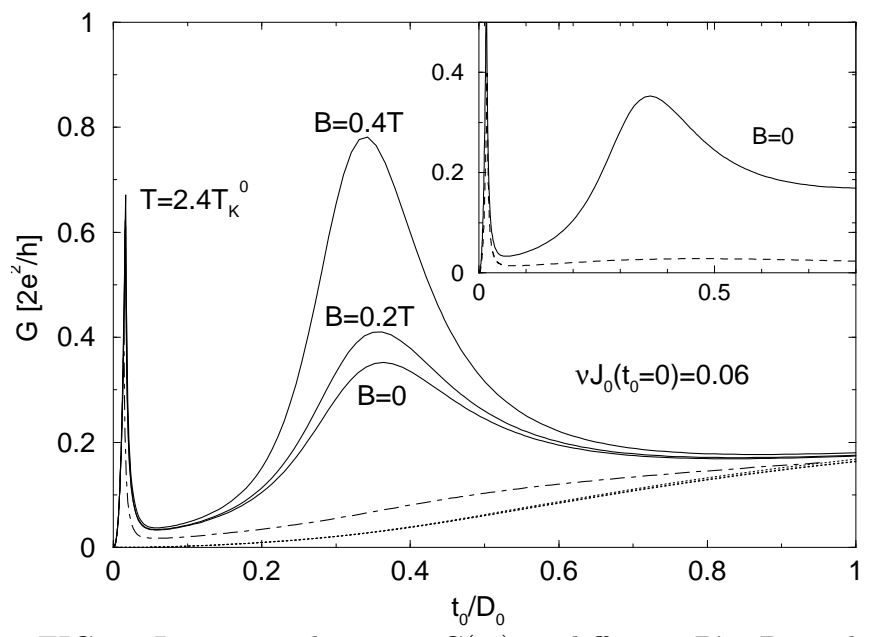

FIG. 2. Linear conductance $G\left(t_{0}\right)$ at different $B$ 's. Dotted lines: cotunneling contributions. Dot-dashed line: $G$ vs $t_{0}$ at $B=0.4 T$ without the long range part of the Coulomb interaction, i.e. for $\mathrm{v}=0$ in (8). For definiteness, we keep $E_{+}=E_{-}$(middle of CB valley) by adjusting $V_{g}$ when varying $t_{0}$, and set $t_{L}=t_{R}$ in Figs. 2-4. Inset: Comparison of full (solid line) with chain (dashed line) model Hamiltonian.

We turn now to a discussion of the linear conductance, $G=G(\Delta \mu=0)$. First, we consider the case $B=0$. At small $t_{0}$ the RG growth of $K$ is weak (due to near cancellation of the trace terms in (10)), and a limit is reached where each spin is strongly coupled with a lead electron. The Kondo effect of the DD, in this case, consists of two independent spin $1 / 2$ Kondo effects for each of the dots separately. At large $t_{0}$, the RG correction to $K$ can be neglected because of a large value of $K_{0}$. The two dot spins are locked into a singlet state in this case, and the lead electrons feel only the potential scattering, slightly enhanced by the RG flow (9) which terminates at $D=K$. At intermediate $t_{0}$, such that $K \sim T_{K}$, the exchange interaction (between the dot spins) and the spin $1 / 2$ Kondo effect compete, and a crossover between the two regimes occurs. At this crossover, the renormalization of $\mathrm{K}$ is comparable with the Kondo temperature $T_{K}$; each of the dot spins couples to both leads. The conductance versus $t_{0}$ shows a peak at $t_{0} \sim \sqrt{U_{H} T_{K}}$ (see Fig. 2). A second peak at larger $t_{0}$ emerges due to the interplay between Kondo correlations and long range Coulomb interaction, see dot-dashed line in Fig. 2. This striking sensitivity of the second peak on the long range Coulomb interaction provides a way of studying the screening effects in coupled quantum dots. As a check we have taken the Hubbard limit (chain Hamiltonian) of our model, and 




FIG. 3. Linear $G$ vs $B$ around $K=0$. The kinks in the dotted-line regions are an artifact of our two stage $\mathrm{RG}$ procedure in the crossover regime and will be smoothened in an exact treatment. The stars denote $B_{*}$ with $K\left(B_{*}, D=D_{0}\right)=0$. Left inset: Cotunneling conductance vs $B$ at small coupling to the leads where Kondo correlations become negligible. Right inset: Renormalized $K$ vs $B$, showing a sharp singlet-triplet transition at low $T\left(\sim T_{0}\right)$.

found good agreement with the exact NRG calculations of Ref. [12]. In inset of Fig. 2 we compare the two models to illustrate the importance of the long range Coulomb interaction. For small $B$ (with $K>0$ ), we find that the second peak in $G\left(t_{0}\right)$ is very sensitive to $B$, see Fig. 2 .

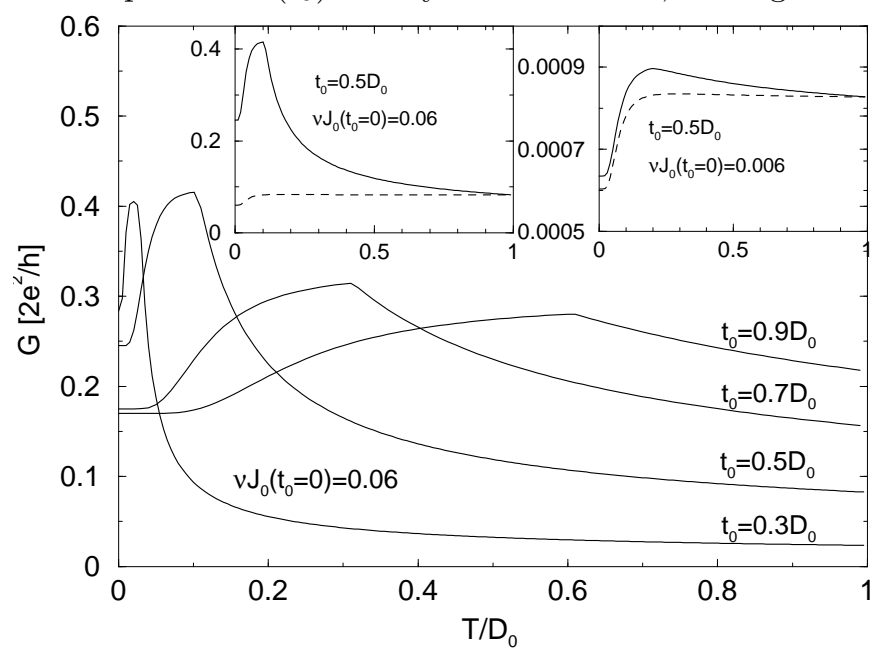

FIG. 4. Linear $G$ vs $T$ for different $t_{0}$. Insets: Comparison of cotunneling (dashed line) and renormalized (solid line) calculations for different couplings to the leads.

At larger $B$, a singlet-triplet transition occurs [15]. Around this point $K=0$, the RG flow (9) terminates at $D=T>|K|$, which leads to a striking RG enhancement of the peak in the cotunneling conductance, see Fig. 3 and left inset. In the singlet regime, for $K>T$, the RG flow terminates at $D=K$, and there is no Kondo limit. Physically, this means that the DD spins are locked into a singlet with exchange energy $K>0$. In the triplet regime, for $-K>T$, we apply a two stage $\mathrm{RG}$ procedure 22] and find a $K$-dependent energy scale for the spin 1 Kondo effect, very much like as in single dots [8,9]. At low $T$, the renormalized $K$ shows a striking dependence on $B$ around $K=0$, see right inset of Fig. 3. Our perturbative RG thus suggests a sharp transition between the two regimes, with a rapidly vanishing spin 1 Kondo temperature when approaching the singlet side. Finally, in Fig. 4 we plot the conductance vs $T$. We see that again the Kondo correlations lead to a pronounced peak in G which occurs at $T=K$, thus providing a further way to access the exchange interaction $K$.

Acknowledgments. We thank G. Burkard, L. Glazman, and M. Pustilnik for discussions. We acknowledge support from the Swiss NSF and DARPA.

[1] L.P. Kouwenhoven, G. Schön, L.L. Sohn, Mesoscopic Electron Transport, NATO ASI Series E, Vol. 345, Kluwer Academic Publishers (1997).

[2] L.I. Glazman, M.E. Raikh, JETP Lett. 47, 452 (1988); T.K. Ng, P.A. Lee, Phys. Rev. Lett. 611768 (1988).

[3] D. Goldhaber-Gordon, et al. Nature (London) 391, 156 (1998).

[4] S.M. Cronenwett, T.H. Oosterkamp, L.P. Kouwenhoven, Science 281, 540 (1998).

[5] J. Schmid, et al. Physica B 256-258, 182 (1998).

[6] T.W. Odom, et al. Science 290, 1549 (2000); J. Nygard, D.H. Cobden, P.E. Lindelof, Nature (London) 408, 342 (2000).

[7] S. Tarucha, et al. Phys. Rev. Lett. 84, 2485 (2000); S. Sasaki, et al. Nature (London) 405, 764 (2000); J. Schmid, et al. Phys. Rev. Lett. 84, 5824 (2000).

[8] M. Pustilnik, L.I. Glazman, Phys. Rev. Lett. 85, 2993 (2000); Phys. Rev. B 64, 045328 (2001).

[9] M. Eto, Y. Nazarov, Phys. Rev. Lett. 85, 1306 (2000); Phys. Rev. B 64, 085322 (2001).

[10] W. Hofstetter, H. Schoeller, cond-mat/0108359.

[11] T. Aono, M. Eto, Phys. Rev. B 63125327 (2001); A. Georges, Y. Meir, Phys. Rev. Lett. 82, 3508 (1999).

[12] W. Izumida, O. Sakai, Phys. Rev. B 62, 10260 (2000).

[13] D. Loss, D.P. DiVincenzo, Phys. Rev. A 57, 120 (1998).

[14] J.M. Kikkawa, D.D. Awschalom, Phys. Rev. Lett. 80, 4313 (1998).

[15] G. Burkard, D. Loss, D.P. DiVincenzo, Phys. Rev. B 59 2070 (1999).

[16] F.R. Waugh et al., Phys. Rev. Lett. 75705 (1995).

[17] J.R. Schrieffer, P.A. Wolff, Phys. Rev. 149, 491 (1966).

[18] P.W. Anderson, J. Phys. C 3, 2436 (1970).

[19] Eqs. (9) hold for arbitrary $t_{\alpha n}$ and can also describe a parallel (Aharonov-Bohm) geometry of the DD.

[20] B.A. Jones, C.M. Varma, Phys. Rev. Lett. 58, 843 (1987).

[21] E.V. Sukhorukov, G. Burkard, D. Loss, Phys. Rev. B 63 125315 (2001).

[22] A.C. Hewson, The Kondo Problem to Heavy Fermions, (Cambridge Univ. Press, 1997). 